\title{
HIGH-DIMENSIONAL SPACE SEARCH SYSTEM FOR MULTI-TOWER CRANE MOTION PLANNING
}

\author{
Minsu Kang ${ }^{1^{*}}$, Hyounseok Moon ${ }^{2}$, and Leenseok Kang ${ }^{3}$ \\ ${ }^{1}$ Department of Electrical Engineering, Seoul National University, Seoul, Korea \\ ${ }^{2}$ Centre for Construction Innovation and Research, University of Teesside, Middelsbrough, UK \\ ${ }^{3}$ Department of Civil Engineering, Gyeongsang National University, Jinju, Korea \\ *Corresponding author (minsuya@gmail.com)
}

\begin{abstract}
The purpose of multi-tower crane motion planning is to find a collision-free construction process for multiple tower cranes operating in a construction site. This problem can be reduced to the path searching problem in highdimensional space and an important amount of research related to the path search algorithm has been published in various fields. In this paper, we analyzed the features of high-dimensional space reduced from the construction site, and we found that the generated space has particular patterns. Therefore, we propose that the previously developed path searching algorithms can easily be optimized to the space. Finally, this study presents an implementation of goal-biased rapidlyexploring random trees, and running the algorithm in the reduced space allows us to obtain the collision-free construction process for multiple tower cranes effectively.
\end{abstract}

Keywords: Multi-Tower Crane, Multi-Robot Path Planning, Rapidly-exploring Random Trees

\section{INTRODUCTION}

Limited size of free space has resulted in the construction projects to build dense and high buildings these days. Moreover, civil engineering projects focus on the speed of construction to meet the demands of people who want to see their buildings as soon as possible. It is true that short construction duration usually has considerable economic benefits. To realize fast construction projects on dense and high buildings, multiple tower cranes are used in the projects. Therefore, position and motion of each tower crane become important to avoid collisions in the construction works. Optimal positions of tower cranes are considered with genetic algorithm in [1], wireless communication system was devised for multiple tower cranes in [2], collision-free motion planning for multiple tower cranes are studied with heuristic approach in [3-4] and virtual simulation method using heuristic search algorithm on multi-tower crane is proposed in [5].

This study introduces that the motion planning problem for multiple tower cranes can be reduced to the path searching problem in high-dimensional space. An important amount of research related to the path search algorithm has been published in the fields of robotics, mathematics, and computer science. However, not much research has been done to develop path-planning methods specifically for multi-tower crane problem. Little research that considered the motions of multiple tower cranes just applied heuristic search algorithm to the problem, but not optimized the algorithms to the problem.

In this paper, we found that the search space reduced from the construction site has particular form of obstacles. Therefore, we propose that the previously developed path searching algorithm can easily be optimized for the multitower crane problem. Based on the rapidly-exploring random trees (RRTs) [6], we present an optimized algorithm. We use the multiple-probabilistic distributions when generating sample node in RRTs to give bias to the goal point, and this allows us to search the collision-free path very effectively in the reduced space. 


\section{PROBLEM REDUCTION}

Multiple tower cranes operating in a construction site can be reduced to a point in a high-dimensional space. We denote the construction site as work space ( $W$-space), and the reduced high-dimensional space as the configuration space ( $C$-space) $[7]$.

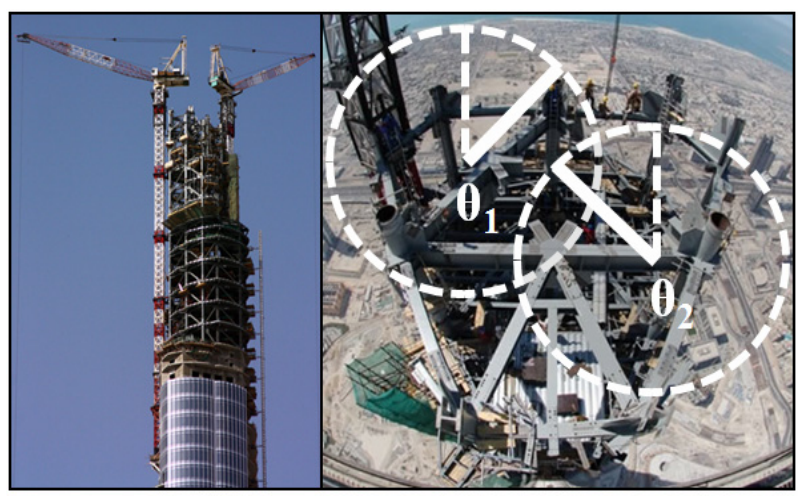

Fig. 1 Two tower cranes working in Burj Dubai tower project.

$W$-space of two tower cranes working in a real construction site is shown in Fig. 1. Dashed circle denotes the working radius of each tower crane. Note that the status of each tower cranes in a construction site can be expressed easily by using their angles. These angles become important information in $C$-space.

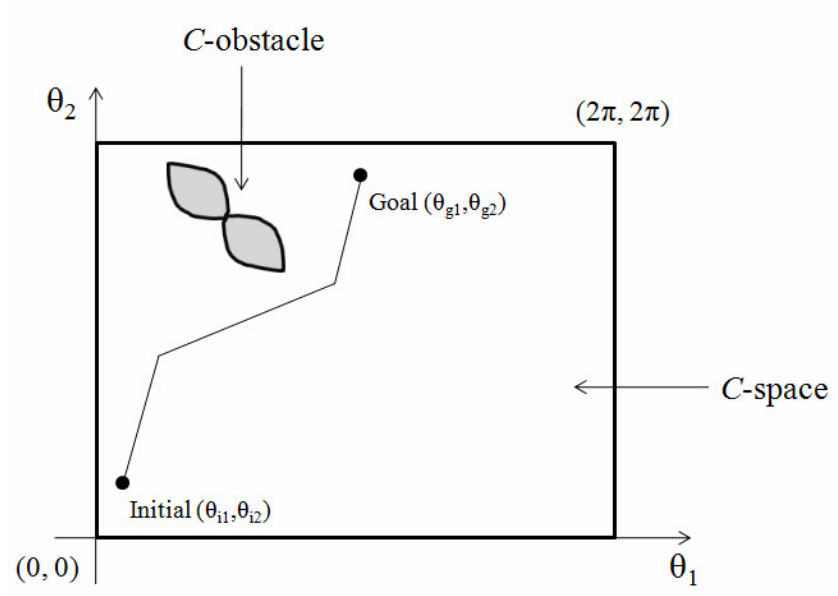

Fig. 2 Motion planning for two tower cranes in $C$-space.

$C$-space of two tower cranes operating in a construction site is shown in Fig. 2. The dimension of $C$-space is equal to the number of tower cranes. Motion planning that realizes the anti-collision work for multiple tower cranes can be solved by searching the anti-collision path from initial point to goal point in $C$-space. Point in the space denotes the status of two tower cranes and the $C$-obstacle is determined by the overlap region of two tower cranes in the $W$-space. By avoiding the $C$-obstacles in $C$-space during the path search, two tower cranes are able to reach the desired state without collision. Note that we are considering two tower cranes simultaneously when we follow the path in $C$-space.

\section{FEATURES OF C-SPACE}

Unlike previous research that applied heuristic algorithm to motion planning problem for multiple tower cranes, we first analyzed the $C$-space reduced from the construction site.

\section{Obstacle density of C-space}

$C$-obstacles in this problem represent the illegal state of tower cranes that occurs the collision. Therefore, overlap regions in the $W$-space generate the $C$-obstacles. Because tower cranes are not placed densely in the construction sites usually, we found that the obstacle density of reduced search space is considerably sparse compared to the density of space usually used in the field of robotics.

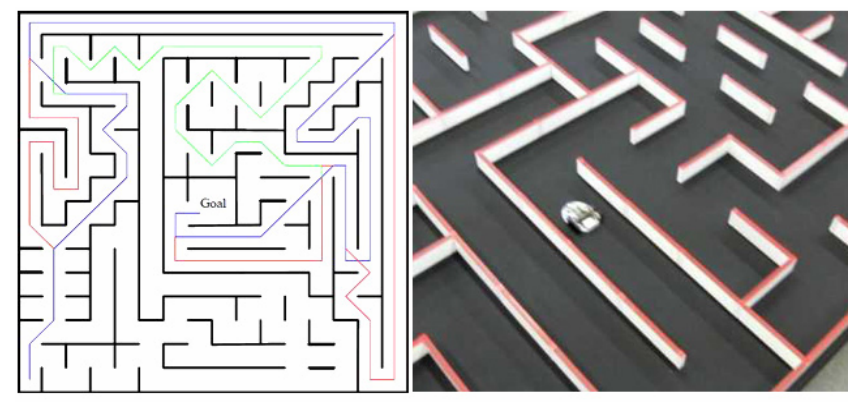

Fig. 3 Example of $C$-space used in the field of robotics.

An example of the dense $C$-space is shown in Fig. 3. Note that form of $W$-space and $C$-space are similar in this case. Because previously developed path searching algorithms are developed to solve such difficult environments, it is inefficient to use the algorithms directly in the multi-tower crane problem. 
Form of C-Obstacle

Overlap region in $W$-space is converted to the obstacles in $C$-space.

Tower Crane 1

Tower Crane 2

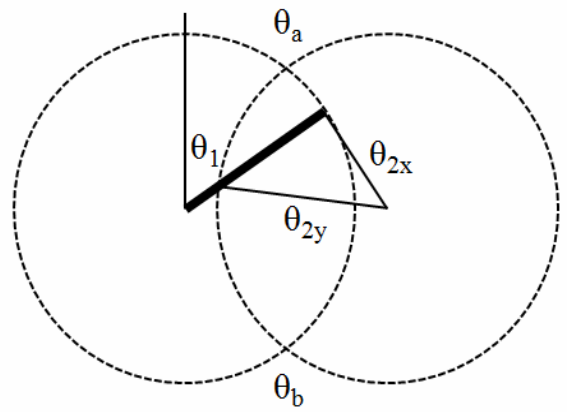

Fig. 4 Overlap region in $W$-space.

Fig. 4 shows the overlap region between two tower cranes. Interested two points are the $\Theta_{a}$ and $\Theta_{b}$. When $\theta_{1}$ moves from $\Theta_{a}$ to $\Theta_{b}$, gap between $\Theta_{2 x}$ and $\Theta_{2 y}$ becomes width of obstacle in $C$-space.

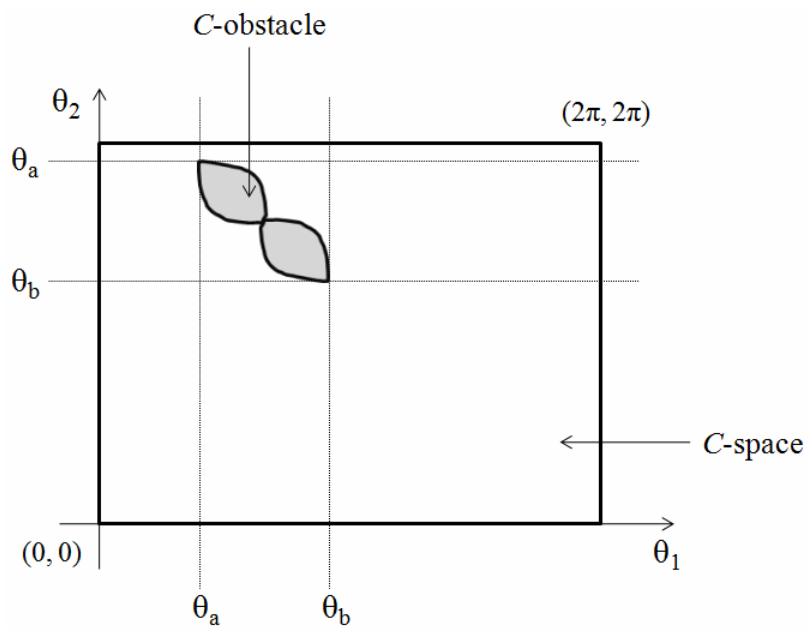

Fig. 5 Converted obstacle in $C$-space.

Converted obstacle in $C$-space is shown in Fig. 5. The obstacle is symmetric about the point $\Theta_{1}=\pi / 2$ and $\Theta_{2}=$ $3 \pi / 2$. Note that both $\Theta_{1}$ and $\theta_{2}$ in Fig. 4 increase clockwise direction. When the number of tower cranes increases, the dimension of $C$-space and $C$-obstacle increases. For example, if a third tower crane is added to the example shown in Fig. 4, the $C$-obstacle shown in Fig. 5 becomes a column in 3-dimensional $C$-space and another
$C$-obstacle will be generated by the additional overlap region. However, $C$-space reduced from the construction site cannot form a difficult environment such as maze shown in Fig. 3. Moreover, note that not all tower cranes operating in a construction site, but tower cranes that have overlap regions have to be considered because they are the candidates that can have collisions.

\section{OPTIMIZED ALGORITHM}

Because the $C$-space used in the multi-tower crane problem has such features, it is easy to optimize the previously developed search algorithm for the space.

\begin{tabular}{|c|c|}
\hline \multicolumn{2}{|c|}{ BUILD_RRT $\left(x_{\text {init }}\right)$} \\
\hline 1 & $T . \operatorname{init}\left(x_{\text {init }}\right)$ \\
\hline 2 & for $k=1$ to $K$ do \\
\hline 3 & $x_{\text {rand }} \leftarrow$ RANDOM_STATE() \\
\hline 4 & $\operatorname{EXTEND}\left(T, x_{\text {rand }}\right)$ \\
\hline 5 & Return $T$ \\
\hline \multicolumn{2}{|c|}{$\overline{\operatorname{EXTEND}}(T, x)$} \\
\hline 1 & $x_{\text {near }} \leftarrow \operatorname{NEAREST}$ _NEIGHBOR $(x, T)$ \\
\hline 2 & if NEW_STATE $\left(x, x_{\text {near }}, x_{\text {new }}, u_{\text {new }}\right)$ then \\
\hline 3 & T.add_vertex $\left(x_{\text {new }}\right)$ \\
\hline 4 & T.add_edge $\left(x_{\text {near }}, x_{\text {new }}, u_{\text {new }}\right)$ \\
\hline 5 & if $x_{\text {new }}=x$ then \\
\hline 6 & Return Reached; \\
\hline 7 & else \\
\hline 8 & Return Advanced; \\
\hline 9 & Return Trapped; \\
\hline
\end{tabular}

Fig. 6 Basic rapidly-exploring random trees.

Algorithm of basic RRTs proposed in [6] is shown in Fig. 6. The algorithm generates random sample and extends the tree to the sample if there is no collision during the expansion. If a collision occurs, tree stops to extend and recursively generate the random sample. RRTs is widely used in the fields of robotics, biology drug design, realtime graphics, and aerospace. Normally RRTs use uniform distribution when generating random sample and doing so allows the RRTs to find solution even in tough $C$-space. 
However, because the $C$-space reduced from the construction site is not a tough environment, it is inefficient to choose the sample by using the uniform distribution. Therefore, we propose to use additional distribution during the sampling.

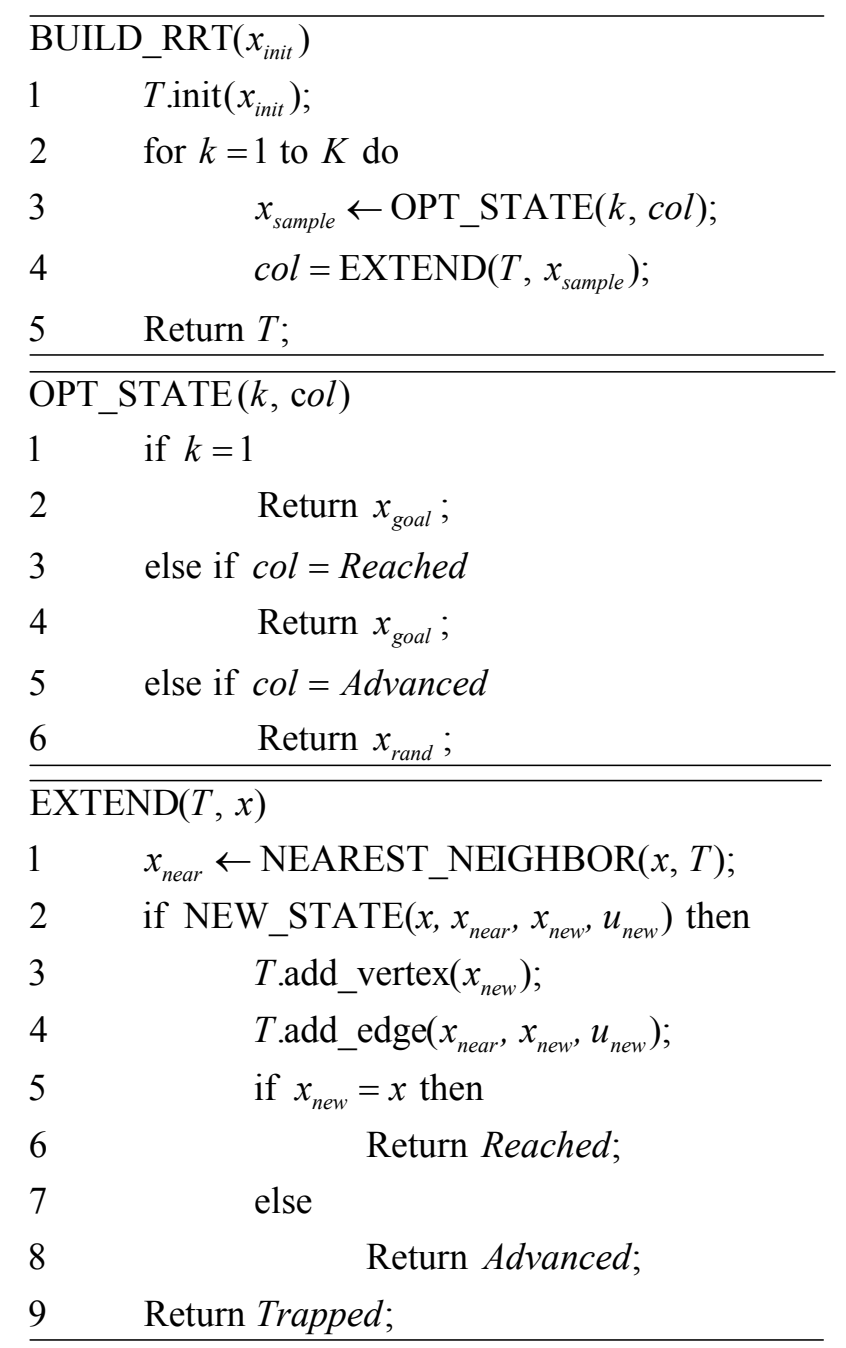

Fig. 7 Rapidly-exploring random trees optimized for multi-tower crane motion planning problem.

The RRTs optimized for the multi-tower crane problem is proposed in Fig. 7. Instead of using RANDOM_STATE function shown in Fig. 6, optimized algorithm generates the sample node with multiple distributions. As shown in OPT_STATE function, the proposed algorithm goes toward the goal at the beginning, and when there is no collision during the expansion. The algorithm generates the sample randomly using uniform distribution only when the collision occurs during the expansion. Therefore, goal- biased RRTs is realized, and these policies are efficient for the $C$-space reduced from the construction site because most of space are free.

\section{EXPERIMENTAL RESULTS}

We tested the optimized RRTs in simulations with various number of tower cranes run on a Intel Core i7 CPU Q740 running at $1.73 \mathrm{GHz}$ with $2.9 \mathrm{~Gb}$ of RAM. In each simulation, basic RRTs was also tested to compare the performance with the optimized one. All simulations were implemented in unoptimized python. Initial and goal position for each tower crane were chosen randomly but no overlap between the positions was guaranteed. In the simulation, each trial was given at most twelve minutes to find a solution.

We visualized the simulation results of two-tower crane problem in Fig. 8 and Fig. 9. Final path solution is included in the tree. Since the proposed RRTs algorithm is optimized for the $C$-space reduced from the construction site, the generated tree during the search process is much simple compared to the tree generated by the basic RRTs algorithm. We also tested the proposed algorithm with tower cranes more than two and the results verified that the proposed algorithm is much stronger for this problem.

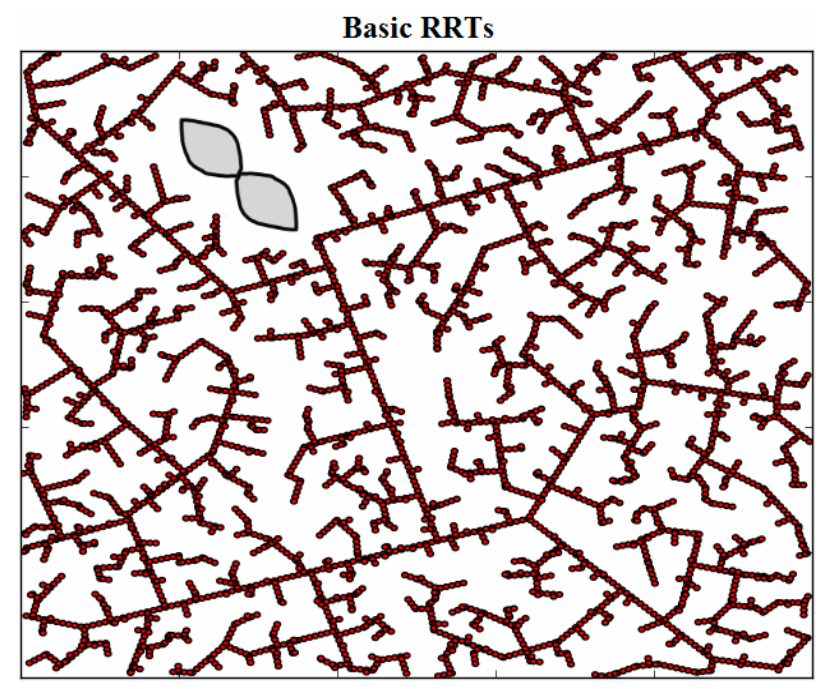

Fig. 8 Tree generated by the basic algorithm. 


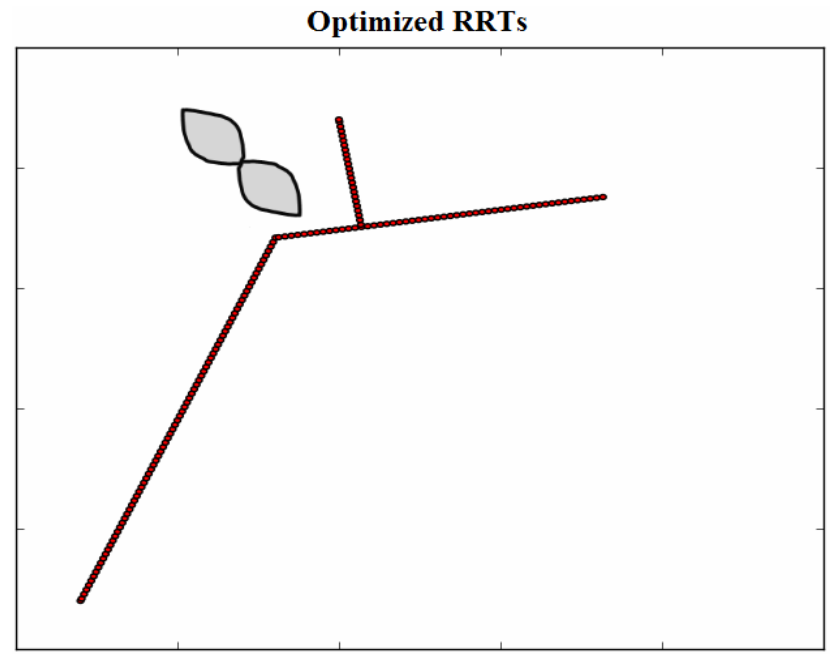

Fig. 9 Tree generated by the optimized algorithm.

Table 1 shows the percent success of each algorithm when the number of tower cranes are two, three and four. Fifty simulations were performed for each case. Simple tree structure generated by the proposed algorithm resulted in the very fast search speed. With more than five tower cranes, most of simulations running basic RRTs were not able to solve the problem, whereas the optimized RRTs were still able to search the space within reasonable time.

Table. 1 Percent success.

\begin{tabular}{|c|c|c|c|}
\hline Dimension & 2 & 3 & 4 \\
\hline Basic & $82 \%$ & $42 \%$ & $22 \%$ \\
\hline Optimized & $100 \%$ & $100 \%$ & $100 \%$ \\
\hline
\end{tabular}

\section{FUTURE WORKS FOR VIRTUAL CONSTRUCTION SYSTEM}

Based on the simulation system for construction project that uses the concept of virtual reality (VR) or augmented reality (AR) [8], the authors are planning to develop a system that can simulate the multi-tower motion planning using the proposed algorithm. Safe motions for multi-tower crane can be given by the algorithm in the system and lots of other factors can be considered based on this information. The system's layout is shown in Fig. 10. Usually, 4D CAD system can simulate the construction activities by time schedule. If an activity uses multi-tower crane, the VR simulation system with this algorithm can visualize the optimized operating routine of tower crane in work space for the activity.

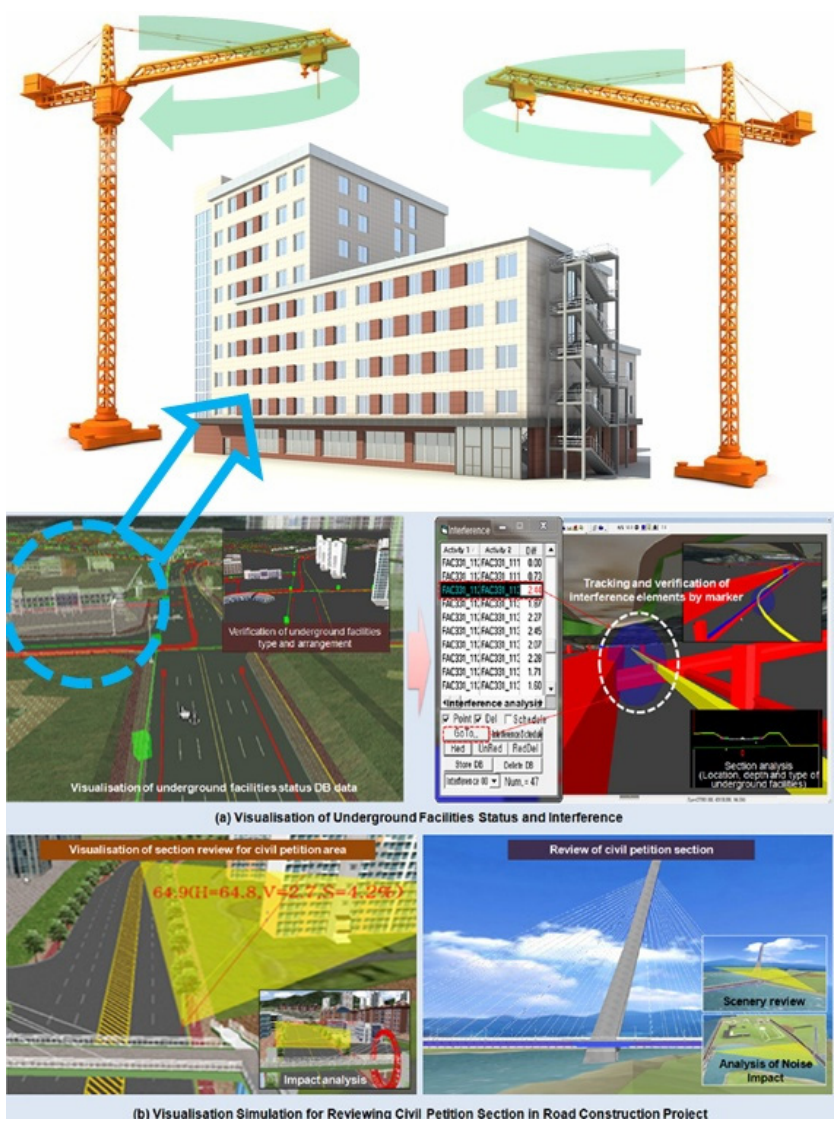

Fig. 10 Concept of virtual construction system linked with the proposed motion planning algorithm.

If the suggested motion planning algorithm is linked with construction simulation system, the functions of building information modeling (BIM) can extend to construction equipment management. And this algorithm also could be a useful tool for simulating the motion of multi-tower cranes that are installed above ground in high-rise buildings.

\section{CONCLUSIONS}

This study introduced the features of $C$-space reduced from the construction site, and proposed the optimized path search algorithm that can effectively solve the motion planning problem for multi-tower crane. As shown in Table 1 , the proposed algorithm is very practical so we expect that the algorithm to be used in many construction projects. By simulating the construction project with a system using the algorithm, construction duration can be reduced 
because tower cranes can be easily located with many overlapping regions. And the overall process of construction can be more efficient because the motions of tower cranes are given. For example, project manager can control the materials to be moved efficiently based on the information.

\section{ACKNOWLEDGEMENT}

The authors would like to thank the Ministry of Construction and Transportation of Korea for financially supporting a part of this research under 2010 R\&D program (Virtual Construction System).

\section{REFERENCES}

[1] C.M. Tam, K.L. Tong, and K.W. Chan, "Genetic algorithm for optimizing supply location around tower crane", Journal of Construction Engineering and Management, pp. 315-321, 2001.

[2] U.K. Lee, K.I, Kang, G.H. Kim, and H.H. Cho, "Improving Tower Crane Productivity Using Wireless Technology", Computer-Aided Civil and Infrastructure Engineering, Volume 21, Issue 8, pp. 594-604, 2006.

[3] P.L. Sivakumar, K. Varghese, and N.R. Babu, "Automated path planning of cooperative crane lifts using heuristic search", Journal of Computing in Civil Engineering, pp.197- 207, 2003.

[4] M.S. Ali, N.R. Babu, and K. Varghese, "Collision free path planning of cooperative crane manipulators using genetic algorithm", Journal of Computing in Civil, pp. 182193, 2005.

[5] S.C. Kang and E. Miranda, "Planning and visualization for automated robotic crane erection processes in construction", Automation in Construction, Vol. 15, pp. 398-414, 2006.

[6] S. M. LaValle and J. J. Kuner, "Randomized kinodynamic planning", Proceedings IEEE International Conference on Robotics and Automation, pp.473-479, 1999.
[7] H. Choset, K. M. Lynch, S. Hutchinson, G. Kantor, W. Burgard, L. E. Kavraki and S. Thrun, "Principles of Robot Motion: Theory, Algorithms, and Implementations", pp.3969, MIT Press, Boston, 2005.

[8] L. S. Kang, H. S. Moon, N. Dawood, M.S. Kang, "Development of methodology and virtual system for optimised simulation of road design data", Automation in Construction, 19(8), pp. 1000-1015, 2010. 